\title{
Physics award acclaims superfluid helium
}

London. The 1996 Nobel prize for physics has been awarded to Douglas Osheroff of Stanford University, California, and to David Lee and Robert Richardson of Cornell University, New York. Their 1971 discovery of superfluidity in the helium isotope ${ }^{3} \mathrm{He}$ spurred two decades of research into the behaviour of macroscopic systems at very low temperatures, at which the effects of quantum mechanics are most evident.

Superfluids are unlike normal fluids. Coffee stirred in a cup eventually comes to rest, its energy dissipated by internal friction. But a superfluid has no internal friction. Consequently, it flows without dissipation, slips easily through cracks too small for normal liquids to penetrate and, under some conditions, can even flow uphill.

Superfluids derive these strange properties from a quantum mechanical effect known as Bose-Einstein condensation, in which a large proportion of the fluid's particles all begin to move in an identical fashion.

Many elements would in principle exhibit superfluidity, but freeze solid before they can be cooled to a sufficiently low temperature. Helium is unique in remaining liquid even as its temperature approaches absolute zero. It is therefore the only element in which the effect occurs. Superfluidity was first discovered in 1938 in liquid ${ }^{4} \mathrm{He}$, the most abundant isotope of helium on Earth.

For many years it was believed that the much rarer isotope ${ }^{3} \mathrm{He}$ would not behave similarly. According to quantum mechanics, particles fall into two classes, 'fermions' and 'bosons', depending on the value of an

\section{IMAGE UNAVAILABLE FOR COPYRIGHT REASONS}

\section{Douglas Osherhoff with apparatus that he uses to study properties of liquid helium.}

attribute called 'spin'. Whereas ${ }^{4} \mathrm{He}$ atoms are bosons, ${ }^{3} \mathrm{He}$ atoms are fermions. And, because only bosons can undergo BoseEinstein condensation, superfluidity in ${ }^{3} \mathrm{He}$ appeared unlikely.

But in 1957 John Bardeen, Leon Cooper and $\mathbf{J}$ Robert Schrieffer showed that, in a

liquid of fermions, any small attractive force between particles would bind them into boson-like pairs, which then could undergo BoseEinstein condensation. This theory explained the low-temperature behaviour of many metals and alloys, in which electrons pair and lead to superconductivity - a close analogue to superfluidity. Its success suggested that superfluidity might occur in ${ }^{3} \mathrm{He}$, the atoms of which attract one David M. Lee (left) and Robert C. Richardson, both of another weakly by van der Waals Cornell University, toast their Nobel success. forces.

"There were many theoretical predictions of a superfluid transition in ${ }^{3} \mathrm{He}$," says Tony Leggett, a theoretical physicist at the University of Illinois, pointing out that some estimates of the transition temperature were as low as $10^{-17} \mathrm{~K}$. But, despite much effort, even by 1970 superfluidity had not been detected in ${ }^{3} \mathrm{He}$.

In 1971, Osheroff was Lee's graduate student, and Richardson was a young faculty member at Cornell. The three were investigating nuclear antiferromagnetism in solid ${ }^{3} \mathrm{He}$ and, according to Lee, made their discovery "more or less by accident".

The group's work required a magnet weighing several tons - a rare commodity among researchers. In autumn 1971, when another group at Cornell needed the magnet, Osheroff decided to test a new refrigeration device that the group had acquired. Helium-3 is unusual in that energy is needed to change it from liquid to solid, a characteristic that can be exploited to cool a mixture of liquid and solid helium to exceedingly low temperatures.

The group devised an experiment in which a solid-liquid mixture was cooled at a constant rate. The experiment went as planned until they saw an unexpected fluctuation in the cooling rate when the temperature reached $2.6 \mathrm{mK}$. Not thinking about superfluidity at the time, the team published a paper suggesting that the effect might

reflect a phase transition in the organization of spins in the solid.

They then carried out a series of nuclear magnetic resonance experiments to isolate the effects of the solid and liquid in the system, and found that several phase transitions were occurring. One was solely in the liquid. Eventually the group began to suspect that they were seeing a BardeenCooper-Schrieffer transition from a normal fluid to a superfluid.

Osheroff says that he still has his laboratory notebook in which the data were recorded. Like many graduate students, he often worked in the middle of the night. An entry at 2.40am on 20 April 1972 reads: "Have discovered the BCS transition in liquid ${ }^{3} \mathrm{He}$ tonight."

Ironically, the paper reporting these results was initially rejected by Physical Review Letters. One referee argued that the system "cannot do what the authors are suggesting it does". But the authors appealed, and the paper was eventually published.

Further experiments by other groups revealed that ${ }^{3} \mathrm{He}$ was far more complex than expected. "Once the experiments started coming out, it soon became clear that theorists had failed to calculate all the really interesting things," says Leggett. For example, ${ }^{3} \mathrm{He}$ has three distinct superfluid phases, while ${ }^{4} \mathrm{He}$ has only one.

Mark Buchanan

\section{Japanese pioneer recalls 'prescient paper'}

Tokyo. Japanese chemist Eiji Osawa was devastated when he saw the paper published in Nature in 1985 (see previous page) describing the discovery of buckminsterfullerene, and comparing its structure to that of a soccer ball.

Fifteen years previously, when at Kyoto University, Osawa had predicted the possible existence of a $\mathrm{C}_{\text {onolecule with a }}$ structure like a soccer ball. But he gave up his work - which was published only in Japanese - when scientists in Japan took little interest and he could see no way of synthesizing the predicted molecule.

"That was the worst day of my life," said Osawa in an interview last year with
Chemical Intelligencer, recalling the appearance of the Nature paper. "This was my baby, and now someone had stolen it."

Osawa, now at Toyohashi University of Technology, applauds the Nobel prizewinners' recognition in that paper of the wider consequences of their discovery.

He has been given credit by Harry Kroto, who in a 1991 paper, describes Osawa's 1970 paper as "a most imaginative and prescient paper". Kroto and his colleagues were unaware of Osawa's work at the time of their 1985 discovery because it was only published in Japanese. Osawa "greatly regrets" not publishing his 1970 work in English. 